\title{
GPs are told to treat with scepticism advice on anti-flu drugs from Public Health England
}

\author{
Deborah Cohen
}

The BMJ

GPs in three English counties have been told to be wary of advice from Public Health England (PHE) to prescribe oseltamivir (Tamiflu) prophylactically to nursing home residents, because of a lack of evidence that the drug is useful in such circumstances.

Paul Roblin, CEO of the medical committee representing GPs in Berkshire, Buckinghamshire, and Oxfordshire, has written to GPs in his area to warn of insufficient evidence for use of the drug in people without symptoms, after GPs raised concerns that they felt bullied by local PHE representatives into prescribing the drug as a preventive measure. Roblin said that guidance from the National Institute for Health and Care Excellence (NICE), which recommends using oseltamivir prophylaxis to prevent influenza in nursing homes, fails to consider unpublished data and reflects publication bias.

At the end of last year the Department of Health gave GPs the go ahead to prescribe antiviral drugs as circulating levels of influenza began to increase. This came after a letter to doctors from Paul Cosford, director of health protection at PHE, which said, "PHE continues to support the early use of antivirals for patients with proven or suspected seasonal influenza who are in high risk groups or who are considerably unwell."

However, Roblin questioned this advice. "Prescribing preventatively to [well people] must surely involve an individual conversation on the risk/benefits ratio and mental capacity assessment as well as renal function. This can be very time consuming," he wrote to local GPs. "If there was a demonstrable benefit in taking the drug then this might be time well spent but the Cochrane review is convincing enough for me," he added.

A Cochrane review-published in April last year and based on full clinical study reports-found no evidence, when oseltamivir was given to elderly people in nursing homes as prophylaxis, that the drug had any effect on influenza symptoms, complications, or hospitalisations. It found, however, a significantly increased risk of gastrointestinal, neuropsychiatric, and renal toxicity. ${ }^{1}$ The Cochrane review also said that Roche, the manufacturer of oseltamivir, had noted that the drug does not prevent influenza infection.

For the treatment indication-that is, when people have influenza - the review found that it shortened the duration of symptoms in the general population, but no separate trials were done in elderly people, although the trial population did include people aged up to 80 . However, in a meeting to discuss Roblin's concerns local officials from NHS England and PHE told him that they were relying on PHE's document, which cites a NICE appraisal published in 2008 about the use of oseltamivir in influenza prophylaxis

This 2008 appraisal included evidence of the prophylactic use of the drug in nursing homes. It relied on a single trial that NICE said showed that oseltamivir had a $92 \%$ protective effect in reducing symptomatic laboratory confirmed influenza $(0.4 \%$ in the placebo group $v 4.4 \%$ in the control group), with an $86 \%$ relative reduction in secondary complications.

It added that, during local outbreaks of influenza-like illness, when there is a high level of certainty that influenza is present, either oseltamivir or zanamivir may be used for post-exposure prophylaxis in patients at risk (regardless of influenza vaccination) who are living in long term residential or nursing homes. This advice is also included in the British National Formulary.

However, after NICE published its 2008 document the Cochrane group found an additional trial on the use of prophylactic oseltamivir in nursing homes that had not been published and not been included in NICE's appraisal. It published details of this trial in its review in April 2014.

The unpublished double blind randomised phase III trial studied oseltamivir use in elderly patients in nursing homes for the prevention of clinical influenza during the flu season. It recruited 380 participants, ran from July 1998 to October 1998, and was a negative trial: it had no effect on the prevention of influenza. However, side effects of receiving the drug were found to include insomnia, vertigo, and diarrhoea.

After the publication of this Cochrane review NICE consulted stakeholders to see whether to review its guidance. The three drug companies that make antivirals all said that the guidance need not be reviewed. The Medicines and Healthcare Products Regulatory Agency and two other groups made no comment, and NICE decided not to revisit the appraisals.

Two of the authors of the Cochrane review-Tom Jefferson, a reviewer for the Cochrane Acute Respiratory Infections Group, and Carl Heneghan, director of Oxford University's Centre for Evidence Based Medicine-have challenged NICE's decision not to review its guidance. They have written to NICE to ask that the appraisal of oseltamivir "be looked into as a matter of urgency, particularly given its effect on clinical decision making."

In a letter on 8 January to David Haslam, chair of NICE, Jefferson and Heneghan complained that the review 
underpinning NICE's appraisal had used "only published studies."

"We have shown the published evidence to be substantially affected by reporting bias," they wrote. They also said that attempts to draw NICE's attention to their latest Cochrane review had been "brushed off" with a "very concerning statement." Aside from the difference in effectiveness outcomes, the Cochrane group had found evidence of major harms, they said.

In November last year a senior NICE official wrote to Jefferson to say that, while NICE appreciated the concerns raised about the safety of technologies, "this is considered to be a matter for the regulators to decide if the potential benefits of these medicines outweigh the risks, and as such, an update of either piece of guidance is not required at this time."

But Jefferson and Heneghan told Haslam that they considered the current position of NICE "incompatible with making evidence based guidance," particularly if the institute "is not prepared to look at the benefit to risk ratio" and instead relies on "expert opinion and economic models that are not based on the transparent evidence base."

In reply Haslam said, "I will certainly look into this and get back to you as soon as possible."

The Cochrane group has also criticised other aspects of PHE's guidance. The PHE document cautions against relying on the
Cochrane group's findings and instead draws on evidence from a Roche funded meta-analysis published last year in The Lancet Respiratory Medicine. This assessed the effect of oseltamivir on mortality in patients admitted to hospital with pandemic influenza.

Jefferson said, "I am confused as to the role of the PHE document in the prescription of oseltamivir to elderly [people] in nursing homes," adding, "The document fails to distinguish treatment and prophylaxis indications (the evidence for the two is quite different) and does not explain the differences between 'seasonal' and 'pandemic' influenza and whether the evidence from our regulatory review is relevant to one, the other, both or neither."

Roblin said, "Neither NICE nor PHE guidance inspires confidence that all the benefits and adverse effects have been adequately assessed in this particular care home scenario, leading GPs to rely more on the Cochrane review, which they trust."

1 Jefferson T, Jones M, Doshi P, Spencer EA, Onakpoya I, Heneghan CJ. Oseltamivir for influenza in adults and children: systematic review of clinical study reports and summary of regulatory comments. BMJ 2014;348:g2545.

Cite this as: BMJ 2015;350:h258

๑ BMJ Publishing Group Ltd 2015 